\title{
Attenuated, Symptom-specific Alteration of Structural Covariance in Patients With Somatic Symptom Disorder
}

Hye Youn Park

Seoul National University of Bundang Hospital

Ye Eun Jang

Seoul National University of Bundang Hospital

Leonard Sunwoo

Seoul National University of Bundang Hospital

In-Young Yoon

Seoul National University of Bundang Hospital

Bumhee Park ( $\sim$ bhpark@ajou.ac.kr)

Ajou University School of Medicine

\section{Research Article}

Keywords: Gray matter volume, Magnetic resonance imaging, Medically Unexplained Symptoms,

Structural covariance, Subtype

Posted Date: April 21st, 2021

DOl: https://doi.org/10.21203/rs.3.rs-428300/v1

License: (c) (1) This work is licensed under a Creative Commons Attribution 4.0 International License.

Read Full License 


\section{Abstract}

This study was performed to investigate altered regional gray matter volume (rGMV) and structural covariance related to somatic symptom disorder (SSD) and its phenotypic subtypes. Additionally, this study examined the relationships of structural alteration with cognitive functioning and clinical symptoms. Forty-three unmedicated patients with SSD and thirty normal controls completed psychological questionnaires and neurocognitive tests, as well as brain magnetic resonance imaging. Voxel-based morphometry and structural covariances were compared between groups and between subgroups within the SSD group. Patients with SSD exhibited attenuated structural covariances in the pallidal-cerebellar circuit, as well as regions in the default mode and sensorimotor network, compared to normal controls. The cerebellar rGMVs were negatively correlated with the severity of somatic symptoms. With respect to cognitive functioning, the rGMVs of the right pallidum, left medial orbitofrontal gyrus, and bilateral supramarginal gyrus were correlated with attentional capacity in SSD patients. In subgroup analyses, patients with somatic pain showed denser structural covariances between the bilateral superior temporal pole and left angular gyrus, the left middle temporal pole and left angular gyrus, and the left amygdala and right inferior orbitofrontal gyrus, while patients with headache and dizziness had greater structural covariance between the right inferior temporal gyrus and right cerebellum. The findings revealed the structural alteration involved in SSD; they also suggested that specific brain regions and networks may contribute to different subtypes of SSD.

\section{Introduction}

Somatic symptom disorder (SSD) as defined by the Diagnostic and Statistical Manual of Mental Disorders, Fifth Edition (DSM-5), ${ }^{1}$ involves excessive and persistent anxiety and somatic disturbances that disrupt activities of daily life. The DSM- 5 specifies pain as a symptom of SSD, but patients may also experience fatigue, palpitations, headache, nausea, indigestion, breathlessness, and dizziness ${ }^{2}$ and these have been classified as discrete symptom dimensions. ${ }^{3}$ Indeed, SSD shows diagnostic overlap within a broad range of other functional syndromes, including fibromyalgia, irritable bowel syndrome, functional neurological disorder, and somatoform dizziness, with diagnoses primarily based on consensus. ${ }^{4}$ These diagnoses are presumed to represent a common disease with different subtypes rather than multiple distinct diseases, where clinical phenotypes, diagnostic criteria, etiopathology, and treatment responses are similar among subtypes. ${ }^{4,5}$ However, there is little concrete evidence to support this perspective. Moreover, some symptoms as chest pain, breathlessness and dizziness, have been understudied compared to somatic pain ${ }^{2}$ and the neural circuits related to specific symptomatic presentation have rarely been investigated. Separate examinations of underlying brain networks for core psychological features of SSD and specific symptoms may produce additional evidence to address the perspectives that SSD represents separate syndromes or subtypes of a single disease.

In the meanwhile, previous neuroimaging studies on somatization disorder, somatoform disorder, and pain disorder, which all fall under SSD in the DSM-5, have revealed altered neural circuitries involved in 
the development of SSD. Patients with SSD have consistently shown changes in the premotor and supplementary motor cortex, middle frontal gyrus (MFG), anterior cingulate cortex (ACC), insula and posterior cingulate cortex (PCC) in both structural and functional neuroimaging studies. ${ }^{6}$ In structural imaging studies of patients with SSD, morphological alterations and gray matter volume (GMV) changes have been observed in the caudate, ACC, MFG, angular gyrus, pituitary, amygdala, hypothalamus, fusiform gyrus, cuneus, inferior frontal gyrus, PCC and cerebellum. ${ }^{6-8}$ Functional studies have also revealed disturbance of functional activity in the default mode network (DMN). ${ }^{9,10}$ Moreover, a recent study found aberrantly increased functional connectivity among the DMN, sensorimotor network (SMN), salience network, and dorsal attention network (DAN) in patients with SSD; these findings suggest that deficits in attention and perception are involved in SSD. ${ }^{11}$

However, such outcomes remain unknown in terms of functional network changes or patterns caused by regional morphological alterations in patients with SSD. Thus, the present study investigated patterns in brain anatomical structures in patients with SSD, by means of structural covariance analysis, which was recently introduced and has not yet been applied in this population. ${ }^{12}$ Structural covariance analysis can capture co-varied morphological characteristics in GMV or cortical thickness across brain areas, and these covariances partially overlap with functional connectivity because they are related to gray matter development and exhibit functional co-activation with neuroplasticity. ${ }^{12}$ Brain areas that co-vary in GMVs may be the part of relevant networks that subserve specific behavioral/cognitive symptoms and functions. ${ }^{13}$ Exploration of such structural covariance in SSD could provide complementary information regarding previous findings of functional magnetic resonance imaging (fMRI) studies; how inter-regional structure are reorganized with morphological changes and which functional connections and symptoms are interrelated. Symptomatic subtypes of SSD may also be differentiated through structural covariance analysis newly.

We hypothesized that 1) patients with SSD would show different structural covariance patterns compared to healthy controls, 2) structural covariance patterns would differ according to phenotypic subtypes of SSD, and 3) structural alteration would be associated with cognitive functioning and clinical symptoms in patients with SSD. To address these hypotheses, we first compared structural covariances between patients with SSD and healthy controls. In comparing patient groups according to phenotypic differences, we also examined structural covaried regions in association with specific symptoms. Additionally, we evaluated the associations between individual regional gray matter volume (rGMV) and neurocognitive performance in SSD, which enable exploration of the involvement of each cognitive function in relation to the structural alteration of brain in patients with SSD.

\section{Results}

Demographic and clinical characteristics of the participants

The demographic and clinical data are presented in Table 1. No significant differences in demographic characteristics were observed between the groups. Patients with SSD had significantly higher scores on 
questionnaires and exhibited poorer performances than the normal controls on the AVLT, CPT, and VFT (Table 1). Finally, all of 22 patients of subtype 1 group had dizziness with additional symptom as headache or concentration difficulties. All of 21 patients of subtype 2 group had musculoskeletal pain including limb pain or back ache or moving pain with additional symptom as pain on another place or tingling sensation. Three patients of subtype 2 group had previously diagnosed with fibromyalgia.

Comparison of structural covariance between SSD and control group

Although the SSD group did not exhibit any significant changes in regional volume, it exhibited disrupted structures in multiple brain regions (i.e., changed structural covariances), compared with normal control group. In the current study, structural covariances, which were centered at the bilateral pallidum and related to the cerebellum, were significantly decreased in the SSD group, compared with normal control group (Fig. 1 and Supplementary table 1; FDR < 0.05-0.2). In detail, the regional volume of the bilateral pallidum showed altered connections with the cerebellar lobes including the bilateral crus $2,7 \mathrm{~b}$, and 8 lobes, as well as the vermis 8 and 9 regions (Fig. 1b, 1c and Supplementary table 1). Reduced covariance was also found between the left Heschl's gyrus and left superior temporal gyrus (STG), the right superior temporal pole (TPsup) and left dorsolateral superior frontal gyrus (SFGdor), and right precuneus and right middle occipital gyrus (MOG) in patients with SSD.

Correlations between rGMVs and clinical variables

Figure 2 shows the associations of psychological/neurocognitive variables with the rGMVs of multiple brain regions after controlling for age, sex, TIV, and BDI-II and BAI scores $(P<0.01$, uncorrected) for each group; 43 significant differences were found between the SSD and normal control groups, and an association study was conducted for regions showing significance. As shown in Figure $2 b$, the SCL-90RSOM scores were negatively correlated with the rGMVs of right cerebellar lobe 3 and bilateral cerebellar lobes 4-5 in patients with SSD. Regarding the cognitive tests, TMT-A response time was negatively correlated with the rGMV of the right pallidum, while the CPT overt response score was negatively correlated with the rGMV of the left medial orbitofrontal gyrus (OFGmed), and the CPT response time was negatively correlated with the rGMV of the bilateral supramarginal gyrus (SMG) in patients with SSD.

Comparison of structural covariance between SSD subgroups

Patients with SSD subtype 2 showed decreased rGMV at the left MFG, left inferior orbitofrontal gyrus (OFGinf), bilateral Rolandic gyri, right Heschl's gyrus, right middle temporal gyrus (MTG), bilateral cerebellar crus $1,2,7 b, 8$, and 9, and left cerebellar region 10, compared to patients with SSD subtype 1 (FDR $<0.2$; Fig. 3a).

Greater structural covariance was detected between the bilateral TPsup and left angular gyrus, the left middle temporal pole (TPmid) and left angular gyrus, and the left amygdala and right OFGinf in patients with SSD subtype 2. In contrast, structural connectivity was greater between the right inferior temporal gyrus (ITG) and right cerebellar crus 2 in patients with SSD subtype 1 (FDR $<0.1-0.2$; Fig. 3b). 


\section{Discussion}

In this study, we found altered structural covariances between cortical and subcortical regions in patients with SSD. Notably, the cerebellar-pallidal circuit showed the largest reduction in structural covariance in patients with SSD. Although the cerebellum has not been considered a central region in SSD, some studies have suggested cerebellar involvement in pain and somatization disorders. ${ }^{9,14,15}$ Other fMRI studies reported that activity in cerebellar lobules was related to pain processing, ${ }^{16,17}$ suggesting that the cerebellar network may be involved in the processing of chronic affective pain. The pallidum, forms a major motor circuit with the cerebellum and recent evidence has suggested that these two regions are closely interconnected and organized into cognitive, affective, and motor systems. ${ }^{18}$ According to a literature review, the pallidum was a key node in subcortical networks underlying somatosensory amplification, which plays a role in somatic symptoms. ${ }^{2}$ Guo et al. ${ }^{19}$ were the first to report decreased functional connectivity of the pallidum in patients with somatization disorder. In line with previous findings, our study suggested that the set of reduced structural links underlying morphological alteration occurred solely at the cerebellar-pallidal circuit which has been known to be associated with clinically relevant dysfunctions in SSD.

Functional imaging studies of SSD have also revealed involvement of various damaged networks, such as the DMN, SMN, salience network, and DAN. ${ }^{11}$ Consistent with these findings, regions showing reduced structural covariance were part of the DMN (TPsup and SFGdor) and SMN (Heschl gyrus and STG) in our study. The DMN is a network that participates in self-referential activity, cognition and emotional processing ${ }^{20}$ thus previous studies have suggested a role for the $D M N$ in the loss of top-down regulation in neural mechanisms of SSD. ${ }^{10,11}$ The SMN is known to participate in sensory-discriminative processing ${ }^{21}$; thus, interactions among the SMN, DMN and salience network appear to be associated with sensory processing, adjusted by attentional and emotional control in SSD. ${ }^{11,22}$ A previous research reported abnormal functional connectivity in the bilateral MOG of patients with SSD and suggested that activity in this region may be associated with aversive experiences and somatic symptoms ${ }^{14}$ and the dorsolateral prefrontal cortex (DLPFC) including SFGdor is thought to subserve top-down attentional control $^{23}$ and the inhibitory control of pain processing. ${ }^{24}$ Although the present results could not explain how these reduced covariances between regions are related to the mechanisms of SSD, results suggest that there are co-varied structural links which were previously demonstrated as core functional networks in SSD. Further studies using combining neuroimaging modalities may clarify how structural and functional network are related and engaged in SSD.

A few studies have investigated the relationships among brain structural alterations, somatic symptoms, and cognitive functioning in patients with SSD. One study reported that a smaller mean GMV of the right MFG was correlated with more severe somatic symptoms and with impaired executive function in patients with somatization disorder. ${ }^{15}$ In the present study, structural alterations in patients with SSD were related to clinical and cognitive dysfunctions. The SCL-90R-SOM scores were negatively correlated with the rGMVs of right cerebellar lobe 3 and left cerebellar lobes 4-5; thus, a smaller GMV of the regional 
cerebellum was associated with more severe somatic symptoms. This result of the involvement of the cerebellum and its association with somatic symptoms in SSD as shown in fibromyalgia ${ }^{25}$ support that there is common neural involvement as well as similar clinical symptoms between these diagnoses.

In the present study, patients with SSD showed significantly poorer performances than the normal controls on the tests of verbal functioning and sustained attention. Positive associations of left OFGmed and bilateral SMG GMV with sustained attentional capacity, as measured by the CPT, were observed in this study. The OFGmed and SMG were shown to be involved in selective attention, ${ }^{26}$ and previous studies demonstrated impaired attention and attentional bias in SSD patients, who tend to be hypervigilant to somatic sensations, rendering them less responsive to external stimuli. ${ }^{27}$ Impairment in these regions could reduce the capacity for sustained attention, as observed in patients with SSD. Correlation analyses indicated that patients with a smaller rGMV for the right pallidum performed worse on the TMT-A, which measures attention and psychomotor speed. Performance on the TMT-A mostly reflects motor skills, which involve basal ganglia-thalamocortical circuits ${ }^{28}$; this may explain the link between TMT-A performance and pallidum volume. In summary, these results suggest that the right pallidum, left OFGmed and SMG may be relevant structural regions related to neurocognitive functioning in patients with SSD; however further studies are needed to determine whether these associations between regions and cognitive functioning in patients of SSD, involve in the mechanisms of SSD.

In our study, patients with pain exhibited lower GMV for the left MFG, left OFGinf, bilateral Rolandic gyrus, right Heschl gyrus, right MTG, and cerebellum, compared to those of patients with dizziness. Moreover, significant structural connectivity was observed between the bilateral TPsup and left angular gyrus, left TPmid and left angular gyrus, and left amygdala and right OFGinf in patients with pain. These regions are mainly involved in the DMN (i.e., the MFG, OFG, MTG, and angular gyrus), salience network (i.e., the amygdala), and SMN (i.e., the Heschl gyrus, Rolandic gyrus, and cerebellum), ${ }^{29}$ which are key damaged functional networks consistently observed in SSD and pain disorders. ${ }^{9,11}$ These regions are known to involve in memory and emotional tagging of sensory perception thus emotional valence to pain inputs may alter the intensity and experience of pain symptoms. ${ }^{30}$ Meanwhile, patients with dizziness exhibited greater structural connectivity between the right ITG and right cerebellum in the dominant hemisphere (with respect to vestibular functioning) in right-handed individuals. The ITG is involved in visual perception, language, and memory ${ }^{31}$; previous studies reported the relationship of the ITG to alexithymia and somatization disorder. ${ }^{19,32}$ According to an fMRI study, the association between subjective dizziness and functional activity of the ITG may reflect increased load on the visual processing region during dizziness. ${ }^{33}$ Increased structural connectivity between the ITG and cerebellum would enhance the perception and memory of dizziness, which might underlie the maladaptive cortical plasticity and memory consolidation seen in patients. We included patients with dizziness in this study, which are relatively understudied symptoms compared to pain in SSD. Furthermore, dizziness has been placed at the interface of functional neurological disorder and SSD; these diagnoses are defined using similar characteristics, and share some pathophysiology. ${ }^{34}$ Our findings suggest that there are specific structural alterations involved in specific symptom presentations in SSD that differ from the neural circuits 
common to core psychological symptoms of SSD. The present results elaborate further studies on subtyping of SSD to better understand for diagnostic clarification and interventions. There have been only few evidences on treatments for SSD that several forms of psychotherapy showed low-to-moderate evidence for efficacy and there has been limited investigations of peripheral interventions. ${ }^{35}$ These results of different involvement of brain regions according to organ symptoms suggest the further studies on peripheral interventions for specific symptom, for example, vestibular rehabilitation for patients with dizziness. This study had several limitations. First, we could not completely rule out effects of depression and anxiety on clinical symptoms or cognitive functioning, although the BDI-II and BAI scores were used as covariates in the analyses. And we did not include emotional and social functional factors which are associated to clinical symptoms thus further researches are needed to examine relationships among these factors. Second, due to the modest sample size we could not perform correlation analyses between clinical variables and imaging data within subgroups. Lastly, this study used a cross-sectional design, so causal relationships between structural alterations in SSD patients and clinical variables cannot be inferred.

Nevertheless, to the best of our knowledge, this preliminary study is the first study to explore structural alterations using structural covariance analysis, and their relationships with organ symptoms of SSD. In particular, we examined specific brain structural covariance in medication-free patients with dizziness, which has rarely been explored during investigations of SSD. Furthermore, previous studies have reported certain cross-cultural differences in somatic symptoms, thus cultural factors should be considered in researches as well as clinical practices of SSD. ${ }^{36}$ Ethno-genic studies have suggested that Asians more frequently exhibit somatic symptoms compared to members of Western cultures, and patients of Asian countries more often complain of breathlessness and dizziness. ${ }^{37}$ The present investigation of brain structural patterns of SSD in this population may also provide additional understanding of cross-cultural specificity of this disorder. This study demonstrated structural alterations in the pallidal-cerebellar circuit, as well as in the DMN and SMN, in patients with SSD. Correlations were observed between rGMVs and clinical/neurocognitive variables in patients with SSD, which suggests the involvement of structural alterations in SSD. Meanwhile, structural alterations as GMV were subtle in patients with SSD despite of definite discomforts from somatic symptoms. This might suggest there are neural alterations at the level of "functional" than definite "structural" disruptions in SSD which are relevant to "dysfunctional" rather than "impaired" states. Our results of alterations in structural covariance not in rGMV may support this and suggest the utility of the structural covariance analysis combined with functional imaging to explore attenuated structural alterations in this populations. Subtype analyses suggested that specific neural networks and brain regions may contribute to the presentation of organ phenotypic symptoms among various somatic symptoms in SSD but this hypothesis should be tested with detailed experiments in further studies. Also, further studies including other SSD subtypes, such as gastrointestinal and cardiopulmonary symptoms, may facilitate the elucidation of brain networks underpinning the subtypes.

\section{Methods}


Subjects

Forty-three patients with SSD who presented to the Dizziness Center or the Pain Clinic in Seoul National University Bundang Hospital, Republic of Korea, and 30 normal controls were recruited from October 2017 to July 2019. The SSD diagnosis was confirmed by consensus between two clinical psychiatrists following clinical interviews based on the Structured Clinical Interview for DSM-5 Disorders-Clinician Version. ${ }^{38}$ The normal controls (healthy adults without psychiatric or medical illnesses) were matched for age and sex to the patients with SSD. The inclusion criteria were: (1) adults aged 20-64 years; (2) confirmed diagnosis of SSD according to the DSM-5 criteria, two or more somatic symptoms; and (3) no use of psychotropic medication within the previous 2 months. The exclusion criteria were: (1) presence of new-onset neurological and/or medical disease with physical symptoms within the past 6 months; (2) presence of any other mental disease, including major depressive disorder, bipolar disorder, a psychotic disorder, an anxiety disorder, a neurocognitive disorder, or a substance use disorder; (3) history of brain injury and/or brain disease; and (4) presence of a non-MRI compatible device or claustrophobia. As depressive and anxiety symptoms are common in patients with SSD, patients with such symptoms below the threshold for a clinical diagnosis were not excluded. All study participants provided their written informed consent. The study methodology was approved by institutional review board of Seoul National University Bundang Hospital (B1710426302), and all procedures were performed according to the ethical principles of the Declaration of Helsinki.

Clinical and neurocognitive measures

After providing an informed consent, all participants were asked to complete the following questionnaires: the Korean version of the Somatization Subscale in Symptom Checklist-90R (SCL-90RSOM) ${ }^{39}$ the Patient Health Questionnaire-15 (PHQ-15), ${ }^{40}$ the Beck Depression Inventory-II (BDI-II) ${ }^{41}$ the Beck Anxiety Inventory (BAI), ${ }^{42}$ the State-Trait Anger Expression Inventory (STAXI), ${ }^{43}$ and the Toronto Alexithymia Scale-20 (TAS-20). ${ }^{44}$ For subgroup comparisons, we recruited the patients of two symptom subgroups according to their main symptoms. Organ subtype classifications, previously suggested for functional somatic syndromes and somatoform disorders were referenced. ${ }^{4}$ According to that classification, patients were sorted into two subtypes: subtype 1 (general symptoms; $n=22$ ), and subtype 2 (musculoskeletal symptoms; $n=21$ ). Additionally, neurocognitive functions were assessed using the Korean version of the Computerized Neurocognitive Function Test (CNT4.0, Maxmedica Inc., Seoul, Korea $)^{45}$ as follows: Auditory Verbal Learning Test (AVLT), the Trail-Making Test (TMT), the Wisconsin Card Sorting Test (WCST), the Digit Span Test, the Continuous Performance Test (CPT) and the Verbal Fluency Test (VFT). We analyzed these measures as well as basic demographics (i.e., age and sex) using independent two sample t-tests (for continuous variables) and chi-square tests (for categorical variables). Statistical analyses were performed using the MATLAB software (Mathworks, Sherborn, MA, USA).

Voxel-based morphometry (VBM) analysis 
All MRI brain scans were obtained using a 3T Philips Achieva (Philips Healthcare, Inc., Best, Netherlands) with an 8-channel SENSE head coil at SNUBH. T1-weighted images were obtained using a fast field-echo three-dimensional imaging sequence with the following parameters: repetition time (TR), $8.1 \mathrm{~ms}$; echo time (TE), $4.6 \mathrm{~ms}$; flip angle (FA), $8^{\circ}$; field-of-view, $240 \times 240 \mathrm{~mm}^{2}$; acquisition matrix, $240 \times 240$; slice thickness, $1 \mathrm{~mm}$; number of excitations, 1; scan time, $4 \mathrm{~m} 7 \mathrm{~s}$.

To extract regional volumes from the entire brain, a VBM analysis was performed using the VBM-DARTEL procedure (SPM12; Wellcome Trust Centre for Neuroimaging, London, UK), ${ }^{46}$ which provides clearer segmentation of tissues and better registration comparing to a previously optimized VBM method. ${ }^{46}$

No motion artifacts or other abnormalities were found on the T1-weighted images, which were inspected by a well-trained physician. The procedure for preprocessing T1-weighted images involved the following steps: (i) manual reorientation to the anterior commissure, (ii) gray matter segmentation based on a standard tissue probability map provided in SPM12, (iii) creation of a study-specific template, spatial normalization of individual images to the DARTEL template, adjustment for volume signal changes during spatial normalization, and ( () spatial smoothing of gray matter partitions using an $8 \mathrm{~mm}$ full-width at half-maximum Gaussian kernel. After preprocessing, rGMV was obtained by averaging the values in each area from 116 regions, which were defined using the Automated Anatomical Labeling Atlas. ${ }^{47}$

Structural covariance analysis

In each group, we conducted partial correlation analyses between the rGMVs of two regions based on cross-sectional VBM data (covariates of age, sex, and total intra-cranial volume [TIV]) as a measure of structural covaried patterns (Fig. 4a). Then, group-level inferences were applied with Fisher's r-to-z transformation. For this, each correlation value were transformed to normal distributed values, $Z_{N C}$ or $Z_{S S D}=0.5 \times[\log (1+R)-\log (1-R)]$, and compared with $Z=\left(Z_{N C}-Z_{S S D}\right) / \sqrt{ }\left[1 /\left(N_{N C}-3-M\right)+1 /\left(N_{S S D}-3-M\right)\right]$, where $N_{\mathrm{NC}}, N_{\mathrm{SSD}}$, and $M$ each represents sample size for each group and the number of covariates used in partial correlation analysis. Each structural covariance was compared between the SSD group and normal controls (Fig. 4b). SSD group were further grouped to subtype 1 and subtype 2; structural covariances and regional volume differences were compared between the two subtypes (Fig. 4b). The rGMV data were correlated with the questionnaire data using partial correlation analysis, including the BDI-II and BAI scores as covariates, as well as age, sex, and the TIV (Fig. 4c).

All statistical analyses were performed using the MATLAB-based custom software (Mathworks, Sherborn, MA, USA). For inference on structural covariance, several thresholds of false discovery rate (FDR) $=0.05$, $0.1,0.15$ and 0.2 were considered significant for addressing multiple-comparison issues (FDR $<0.05$ for significance). ${ }^{48}$ FDR thresholding controls the expected proportion of false positives only among brain areas exhibiting significant values. ${ }^{48}$ FDR control levels in the range 0.1-0.2 are considered practically acceptable, and several neuroimaging studies have applied them. ${ }^{49,50}$

\section{Declarations}




\section{Acknowledgements}

The present study was supported by the National Research Foundation of Korea (NRF) grant funded by the Korea government (NRF-2017R1C1B2012733).

\section{Disclosure Statement}

All authors have no conflicts of interest to declare.

\section{Author Contributions}

H.Y.P designed this study and mainly wrote the original draft. Y.E.J. and L.S collected the data and performed the analysis of data. I.Y.Y. designed the study and reviewed the manuscript. B. P. mainly performed the analysis of data, wrote and edited the manuscript.

\section{References}

1. American Psychiatric Association. Diagnostic and statistical manual of mental disorders (DSM-5®). American Psychiatric Association Publishing. https://doi.org/10.1176/appi.books.9780890425596.x00diagnosticclassification (2013).

2. Perez, D. L., Barsky, A. J., Vago, D. R., Baslet, G. \& Silbersweig, D. A. A neural circuit framework for somatosensory amplification in somatoform disorders. J. Neuropsychiatry Clin. Neurosci.27, e40e50. https://doi.org/10.1176/appi.neuropsych.13070170 (2015).

3. Kroenke, K., Spitzer, R. L. \& Williams, J. B. The PHQ-15: validity of a new measure for evaluating the severity of somatic symptoms. Psychosom. Med.64, 258-266. https://doi.org/10.1097/00006842200203000-00008 (2002).

4. Fink, P. \& Schröder, A. One single diagnosis, bodily distress syndrome, succeeded to capture 10 diagnostic categories of functional somatic syndromes and somatoform disorders. J. Psychosom. Res.68, 415-426. https://doi.org/10.1016/j.jpsychores.2010.02.004 (2010).

5. Rief, W. \& Broadbent, E. Explaining medically unexplained symptoms-models and mechanisms. Clin. Psychol. Rev.27, 821-841. https://doi.org/10.1016/j.cpr.2007.07.005 (2007).

6. Boeckle, M., Schrimpf, M., Liegl, G. \& Pieh, C. Neural correlates of somatoform disorders from a metaanalytic perspective on neuroimaging studies. Neurolmage Clin.11, 606-613. https://doi.org/10.1016/j.nicl.2016.04.001 (2016).

7. Yildirim, H., Atmaca, M., Sirlier, B. \& Kayali, A. Pituitary volumes are reduced in patients with somatization disorder. Psychiatry Investig.9, 278. https://doi.org/10.4306/pi.2012.9.3.278 (2012).

8. Delvecchio, G. et al. The neuroanatomy of somatoform disorders: A magnetic resonance imaging study. Psychosomatics60, 278-288. https://doi.org/10.1016/j.psym.2018.07.005 (2019).

9. Wang, H. et al. Clinical significance of increased cerebellar default-mode network connectivity in resting-state patients with drug-naive somatization disorder. Medicine95, e4043. 
https://doi.org/10.1097/md.0000000000004043 (2016).

10. Su, Q. et al. Dissociation of regional activity in default mode network in medication-naive, firstepisode somatization disorder. PLoS One9, e99273. https://doi.org/10.1371/journal.pone.0099273 (2014).

11. Kim, S. M., Hong, J. S., Min, K. J. \& Han, D. H. Brain Functional Connectivity in Patients With Somatic Symptom Disorder. Psychosom. Med.81, 313-318. https://doi.org/10.1097/psy.0000000000000681 (2019).

12. Alexander-Bloch, A., Giedd, J. N. \& Bullmore, E. Imaging structural co-variance between human brain regions. Nat. Rev. Neurosci.14, 322-336. https://doi.org/10.1038/nrn3465 (2013).

13. Zielinski, B. A., Gennatas, E. D., Zhou, J. \& Seeley, W. W. Network-level structural covariance in the developing brain. Proc. Natl. Acad. Sci.107, 18191-18196. https://doi.org/10.1073/pnas.1003109107 (2010).

14. Li, Q. et al. Altered regional brain function in the treatment-naive patients with somatic symptom disorder: a resting-state fMRI study. Brain and behavio/6, e00521. https://doi.org/10.1002/brb3.521 (2016).

15. Li, R. et al. Bidirectional causal connectivity in the cortico-limbic-cerebellar circuit related to structural alterations in first-episode, drug-naive somatization disorder. Front. Psychiatry 9, 162. https://doi.org/10.3389/fpsyt.2018.00162 (2018).

16. Helmchen, C., Mohr, C., Erdmann, C., Petersen, D. \& Nitschke, M. F. Differential cerebellar activation related to perceived pain intensity during noxious thermal stimulation in humans: a functional magnetic resonance imaging study. Neurosci. Lett. 335, 202-206. https://doi.org/10.1016/s03043940(02)01164-3 (2003).

17. Moulton, E. A., Schmahmann, J. D., Becerra, L. \& Borsook, D. The cerebellum and pain: passive integrator or active participator? Brain Res. Rev.65, 14-27. https://doi.org/10.1016/j.brainresrev.2010.05.005 (2010).

18. Bostan, A. C. \& Strick, P. L. The basal ganglia and the cerebellum: nodes in an integrated network. Nat. Rev. Neurosci. 19, 338-350. https://doi.org/10.1038/s41583-018-0002-7 (2018).

19. Guo, W. et al. Anatomical distance affects cortical-subcortical connectivity in first-episode, drug-naive somatization disorder. J. Affect Disord.217, 153-158. https://doi.org/10.1016/j.jad.2017.04.008 (2017).

20. Raichle, M. E. The brain's default mode network. Annu. Rev. Neurosci.38, 433-447. https://doi.org/10.1146/annurev-neuro-071013-014030 (2015).

21. Seifert, F. \& Maihöfner, C. Functional and structural imaging of pain-induced neuroplasticity. Curr. Opin. Anaesthesiol.24, 515-523. https://doi.org/10.1097/aco.0b013e32834a1079 (2011).

22. Zhao, Z. et al. Altered resting-state intra-and inter-network functional connectivity in patients with persistent somatoform pain disorder. PloS one12, e0176494. https://doi.org/10.1371/journal.pone.0176494 (2017). 
23. Wiech, K., Ploner, M. \& Tracey, I. Neurocognitive aspects of pain perception. Trends. Cogn. Sci.12, 306313. https://doi.org/10.1016/j.tics.2008.05.005 (2008).

24. Seminowicz, D. A. \& Moayedi, M. The dorsolateral prefrontal cortex in acute and chronic pain. J. Pain 18, 1027-1035. https://doi.org/10.1016/j.jpain.2017.03.008 (2017).

25. Kim, H. et al. Fibromyalgia is characterized by altered frontal and cerebellar structural covariance brain networks. Neuroimage Clin.7, 667-677. https://doi.org/10.1016/j.nicl.2015.02.022 (2015).

26. Yoncheva, Y. N., Zevin, J. D., Maurer, U. \& McCandliss, B. D. Auditory selective attention to speech modulates activity in the visual word form area. Cereb. Cortex20, 622-632. https://doi.org/10.1093/cercor/bhp129 (2010).

27. Rief, W. \& Isaac, M. The future of somatoform disorders: somatic symptom disorder, bodily distress disorder or functional syndromes? Curr. Opin. Psychiatry27, 315-319. https://doi.org/10.1097/yco.0000000000000089 (2014).

28. DeLong, M. \& Wichmann, T. Changing views of basal ganglia circuits and circuit disorders. Clin. EEG Neurosci. 41, 61-67. https://doi.org/10.1177/155005941004100204 (2010).

29. Xiang, J. et al. Graph-based network analysis of resting-state fMRI: test-retest reliability of binarized and weighted networks. Brain Imaging Behav. 14, 1361-1372. https://doi.org/10.1007/s11682-01900042-6 (2020).

30. Blaizot, X. et al. The human parahippocampal region: I. Temporal pole cytoarchitectonic and MRI correlation. Cereb. Cortex 20, 2198-2212. https://doi.org/10.1093/cercor/bhp289 (2010).

31. Hamamé, C. M. et al. Reading the mind's eye: online detection of visuo-spatial working memory and visual imagery in the inferior temporal lobe. Neuroimage59, 872-879.

https://doi.org/10.1016/j.neuroimage.2011.07.087 (2012).

32. Su, Q. et al. Increased functional connectivity strength of right inferior temporal gyrus in first-episode, drug-naive somatization disorder. Aust. N. Z. J. Psychiatry49, 74-81. https://doi.org/10.1177/0004867414553949 (2015).

33. Roberts, R. et al. Functional neuroimaging of visuo-vestibular interaction. Brain Struct. Funct.222, 2329-2343. https://doi.org/10.1007/s00429-016-1344-4 (2017).

34. Bègue, I., Adams, C., Stone, J. \& Perez, D. L. Structural alterations in functional neurological disorder and related conditions: a software and hardware problem? Neuroimage Clin.22, 101798. https://doi.org/10.1016/j.nicl.2019.101798 (2019).

35. Henningsen, P. Management of somatic symptom disorder. Dialogues Clin. Neurosci.20, 23-31. https://doi.org/10.31887/dcns.2018.20.1/phenningsen (2018).

36. Ma-Kellams, C. Cross-cultural differences in somatic awareness and interoceptive accuracy: a review of the literature and directions for future research. Front. Psychol.5, 1379. https://doi.org/10.3389/fpsyg.2014.01379 (2014).

37. Grover, S. \& Ghosh, A. Somatic symptom and related disorders in Asians and Asian Americans. Asian J. psychiatr.7, 77-79. https://doi.org/10.1016/j.ajp.2013.11.014 (2014). 
38. First, M. B., Williams, J. B., Karg, R. S. \& Spitzer, R. L. Structured clinical interview for DSM-5 disorders: SCID-5-CV clinician version. American Psychiatric Association Publishing, (2016).

39. Kim, K., Kim, J. \& Won, H. Korean manual of symptom checklist-90-revision. Seoul: Jung Ang Juk Sung Publisher, 8-10 (1984).

40. Han, C. et al. Psychometric properties of the Patient Health Questionnaire-15 (PHQ-15) for measuring the somatic symptoms of psychiatric outpatients. Psychosomatics50, 580-585. https://doi.org/10.1016/s0033-3182(09)70859-x (2009).

41. Lim, S.-Y. et al. The validation study of Beck Depression Scale 2 in Korean version. Anxiety and mood7, 48-53. https://doi.org/10.1037/t05261-000 (2011).

42. Yook, S. \& Kim, Z. A clinical study on the Korean version of Beck Anxiety Inventory: comparative study of patient and non-patient. Korean J. Clin. Psychol.16, 185-197 (1997).

43. Chon, K.-K., Hahn, D.-W., Lee, C.-H. \& Spielberger, C. Korean adaptation of the state-trait anger expression inventory: anger and blood pressure. Korean J. Health Psychol.2, 60-78 (1997).

44. Lee, Y., Rim, H. \& Lee, J. Development and validation of a Korean version of the 20-item Toronto Alexithymia Scale (TAS-20K). J. Korean Neuropsychiatr. Assoc.35, 888-899 (1996).

45. Kwon, J. S., Lyoo, I. K., Hong, K. S., Yeon, B. K. \& Ha, K. S. Development and standardization of the computerized memory assessment for Korean adults. J. Korean Neuropsychiatr. Assoc.41, 347-362 (2002).

46. Ashburner, J. A fast diffeomorphic image registration algorithm. J. Neuroimage 38, 95-113. https://doi.org/10.1016/j.neuroimage.2007.07.007 (2007).

47. Tzourio-Mazoyer, N., et al. Automated anatomical labeling of activations in SPM using a macroscopic anatomical parcellation of the MNI MRI single-subject brain. Neuroimage15, 273-289. https://doi.org/10.1006/nimg.2001.0978 (2002).

48. Genovese, C. R., Lazar, N. A. \& Nichols, T. Thresholding of statistical maps in functional neuroimaging using the false discovery rate. Neuroimage15, 870-878. https://doi.org/10.1006/nimg.2001.1037 (2002).

49. Gordon, B. A. et al. Cross-sectional and longitudinal atrophy is preferentially associated with tau rather than amyloid $\beta$ positron emission tomography pathology. Alzheimers Dement.10, 245-252. https://doi.org/10.1016/j.dadm.2018.02.003 (2018).

50. Jung, K.-I. et al. Cerebellar Gray Matter Volume, Executive Function, and Insomnia: Gender Differences in Adolescents. J. Scientific reports9, 1-9. https://doi.org/10.1038/s41598-018-37154-w (2019).

\section{Tables}

Table 1. Demographic and clinical characteristics, and results of neurocognitive tests 


\begin{tabular}{|c|c|c|c|c|}
\hline & $\begin{array}{l}\text { SSD } \\
(N=43)\end{array}$ & $\begin{array}{l}\text { Normal Control } \\
(\mathrm{N}=30)\end{array}$ & & \\
\hline & $\begin{array}{l}\text { Mean (SD) or } \\
\text { ratio }\end{array}$ & $\begin{array}{l}\text { Mean (SD) or } \\
\text { ratio }\end{array}$ & Statistics & $p$ \\
\hline \multicolumn{5}{|l|}{ Demographic variables } \\
\hline Age, y & $47.27(11.43)$ & $45.87(9.22)$ & $t=0.56$ & .57 \\
\hline Sex, Male/Female & $13 / 30$ & $9 / 21$ & $\begin{array}{l}\chi^{2}= \\
0.0005\end{array}$ & .98 \\
\hline \multicolumn{5}{|l|}{ Clinical variables } \\
\hline SCL-90-R-SOM & $13.65(7.83)$ & $3.10(3.25)$ & $t=6.96$ & $.001 * * *$ \\
\hline PHQ-15 & $13.07(4.95)$ & $3.03(2.07)$ & $t=10.45$ & $<.001^{\star * \star}$ \\
\hline BDI-II & $15.76(8.33)$ & $3.66(3.82)$ & $t=7.41$ & $.001^{<* *}$ \\
\hline BAI & $20.76(13.43)$ & $2.87(2.95)$ & $t=7.16$ & $<.001^{\star \star \star *}$ \\
\hline STAXI-state & $12.69(5.08)$ & $10.20(0.48)$ & $t=2.68$ & $.009 * \star$ \\
\hline STAXI-trait & $18.79(5.15)$ & $15.30(4.27)$ & $t=3.05$ & $.003^{* *}$ \\
\hline TAS-20 & $53.39(10.81)$ & $39.76(8.64)$ & $t=5.74$ & $.001^{* * *}$ \\
\hline TIV (litres) & $1.38(0.10)$ & $1.37(0.11)$ & $t=0.34$ & .73 \\
\hline \multicolumn{5}{|l|}{ Neurocognitive tests } \\
\hline Auditory Verbal Learning Test & $54.04(7.72)$ & $58.23(5.88)$ & $t=-2.50$ & $.015^{\star}$ \\
\hline $\begin{array}{l}\text { Trail Making Test -A response time } \\
\text { (sec) }\end{array}$ & $25.55(9.82)$ & $22.80(7.53)$ & $t=1.29$ & .20 \\
\hline $\begin{array}{l}\text { Trail Making Test-B response time } \\
(\mathrm{sec})\end{array}$ & $44.83(18.70)$ & $42.10(15.58)$ & $t=0.65$ & .51 \\
\hline Wisconsin Card Sorting Test & $4.83(1.75)$ & $4.96(1.65)$ & $t=-0.32$ & .75 \\
\hline Digit Span Test- forward & $7.49(1.01)$ & $7.65(0.84)$ & $t=-0.71$ & .47 \\
\hline Digit Span Test- backward & $5.79(1.49)$ & $6.13(1.07)$ & $t=-1.07$ & .28 \\
\hline Visual CPT- correct & $134.30(1.75)$ & $135.00(0.00)$ & $t=-2.18$ & $.033^{*}$ \\
\hline Visual CPT- overt & $1.41(2.42)$ & $0.63(0.71)$ & $t=1.72$ & .090 \\
\hline
\end{tabular}




\begin{tabular}{|lllll|}
\hline Visual CPT- response time (sec) & $0.39(0.05)$ & $0.37(0.02)$ & $\mathrm{t}=1.55$ & .12 \\
\hline Verbal Fluency Test & $34.02(10.59)$ & $35.93(10.43)$ & $\mathrm{t}=-0.76$ & .44 \\
\hline Verbal Fluency Test- repeat & $1.27(1.50)$ & $0.63(0.92)$ & $\mathrm{t}=2.09$ & $.040^{*}$ \\
\hline
\end{tabular}

SSD, somatic symptom disorder; SD, standard deviation; SCL-90-R-SOM, Somatization subscale from Symptom Checklist-90-Revised; PHQ-15, Patient Health Questionnaire-15; BDI-II, Beck Depression Inventory-II; BAI, Beck Anxiety Inventory; STAXI-state, State-Trait Anger Expression Inventory-state; STAXItrait, State-Trait Anger Expression Inventory-trait; TAS-20, Toronto Alexithymia Scale-20; TIV, total intracranial volume; $\mathrm{CPT}$, Continuous Performance Test.

All $p$ values are calculated with independent $t$ test (degree of freedom $=71$ for all, 70 for Trail Making TestA) or $\chi^{2}$ test.

${ }^{*} \mathrm{p}<.05, * * \mathrm{p}<.01, * \star \star \mathrm{p}<.001$

\section{Figures}


a

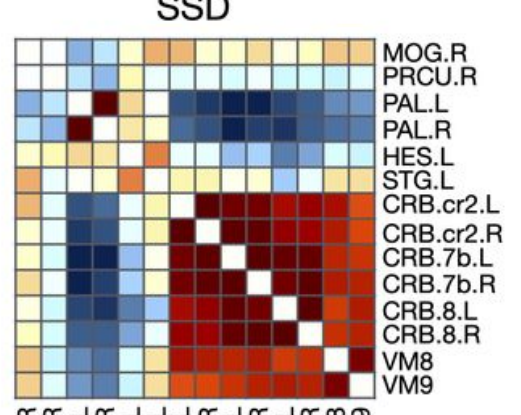

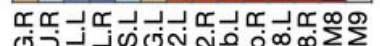

ஸ்่ப்

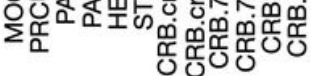
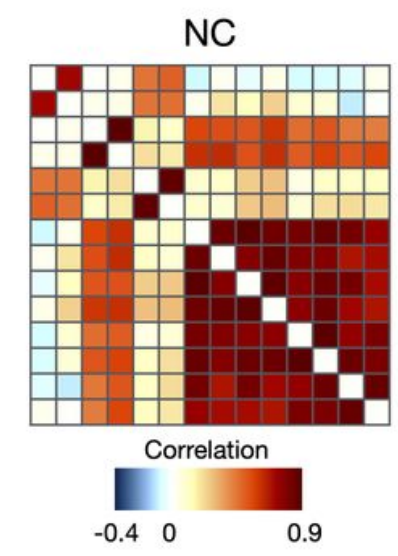

b

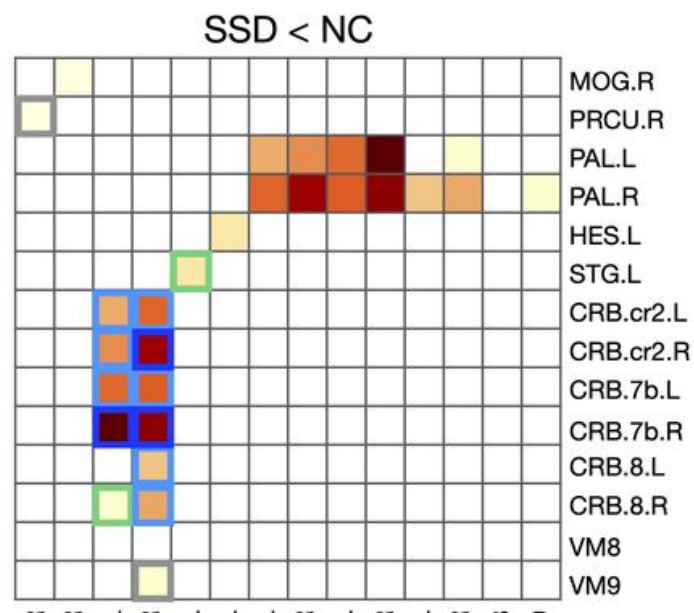

ஊ

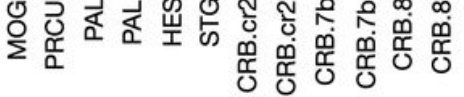
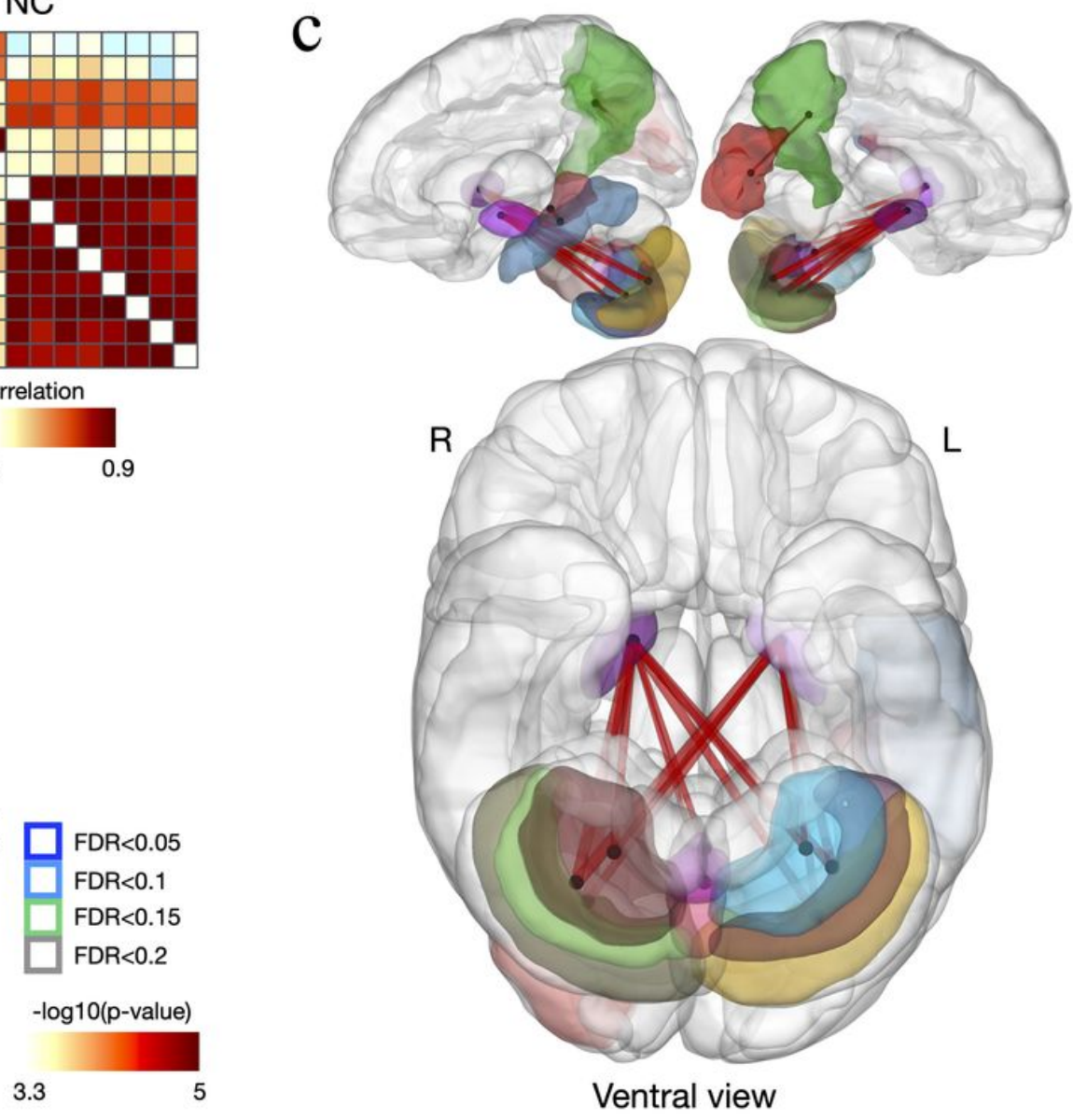

\section{Figure 1}

Disrupted structural covariance connectivity in patients with SSD. (a) Group-averaging structural covariance connectivity for SSD and NC group. (b) Reduced structural covariances and relating regions in patients with SSD and (c) their 3D rendering. SSD somatic symptom disorder; NC normal control; MOG middle occipital gyrus; PRCU precuneus; PAL pallidum; HES Heschl's gyrus; STG superior temporal gyrus; CRcr2 cerebellar crus 2; CR7b cerebellar lobe 7b; CR8 cerebellar lobe 8; VM8 vermis 8; VM 9 vermis 9; L left; $\mathrm{R}$ right. 
a

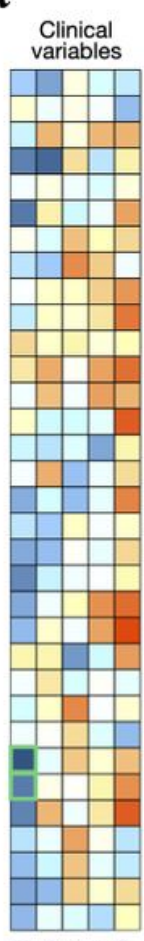

SSD

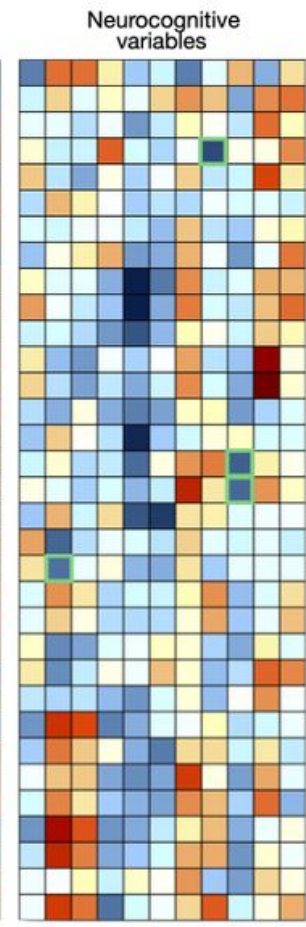

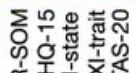

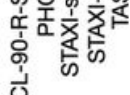

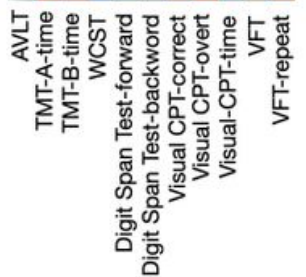

NC
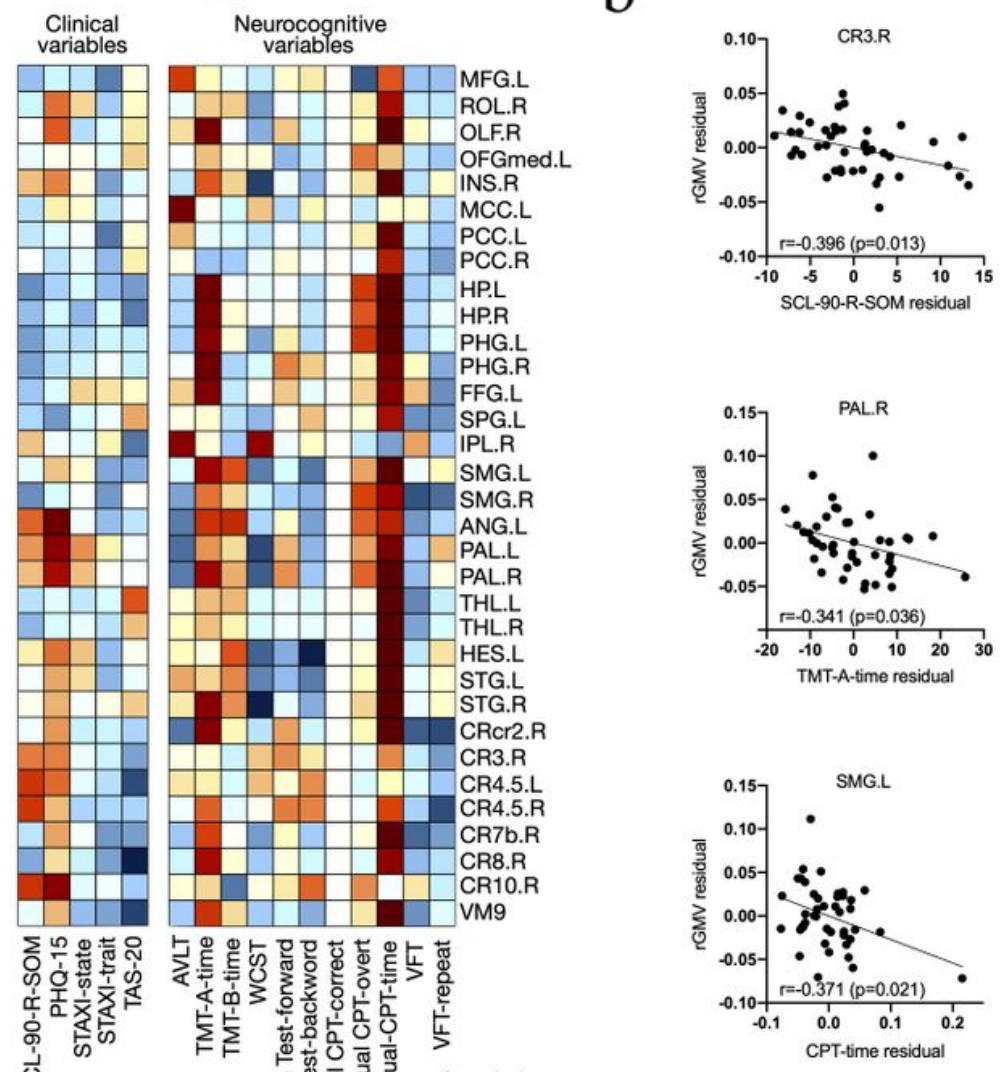
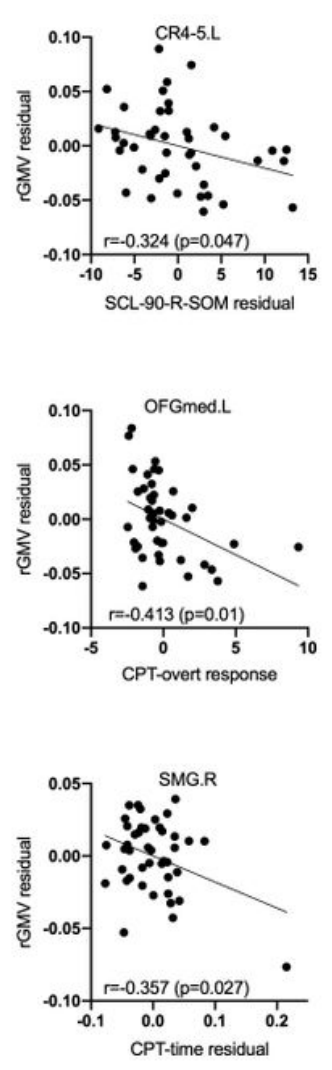

Figure 2

(a) Associations between clinical/neurocognitive variables and rGMVs in the whole brain for each group and (b) remarkable negative correlations among them, presented as scatter plots, are marked as green boxes in (a). rGMV regional gray matter volume; SSD somatic symptom disorder; NC normal control; MFG middle frontal gyrus; ROL Rolandic gyrus; OLF olfactory cortex; OFGmed medial orbitofrontal gyrus; INS insula; MCC middle cingulate cortex; PCC posterior cingulate cortex; HP hippocampus; PHG parahippocampal gyrus; FFG fusiform gyrus; SPG superior parietal gyrus; IPL inferior parietal lobule; SMG supramarginal gyrus; ANG angular gyrus; PAL pallidum; THL thalamus; HES Heschl's gyrus; STG superior temporal gyrus; CRcr2 cerebellar crus 2; CR3 cerebellar lobe 3; CR4.5 cerebellar lobes 4-5; CR7b cerebellar lobe 7b; CR8 cerebellar lobe 8; CR10 cerebellar lobe 10; VM9 vermis 9; L left; R right; SCL-90-R-SOM Somatization subscale from Symptom Checklist-90-Revised; PHQ-15 Patient Health Questionnaire-15; STAXI State-Trait Anger Expression Inventory; TAS-20 Toronto Alexithymia Scale-20; AVLT Auditory Verbal Learning Test; TMT Trail-Making Test; WCST Wisconsin Card Sorting Test; CPT Continuous Performance Test; VFT Verbal Fluency Test. 

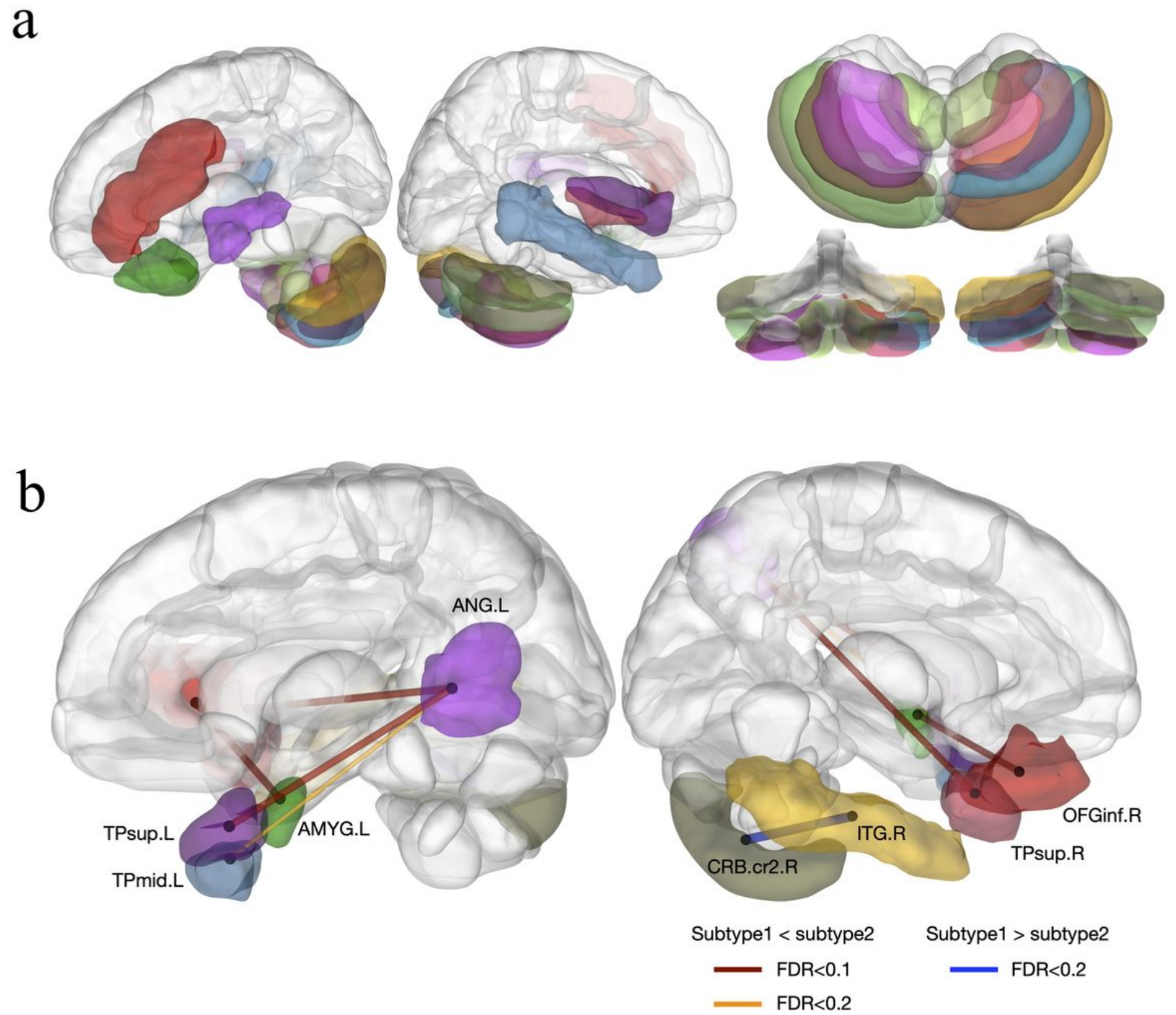

\section{Figure 3}

Differences in regional volumes and structural covariance networks between SSD subtypes. (a) Regional volume difference was reduced in subtype 2 , compared to subtype 1 (FDR $<0.2$ ). (b) Different structural covariance patterns between the two subtypes (FDR < 0.2). TPsup superior temporal pole; AMYG amygdala; ANG angular gyrus; CRcr2 cerebellar crus 2; ITG inferior temporal gyrus; OFGinf inferior orbitofrontal gyrus; L left; R right. 
a
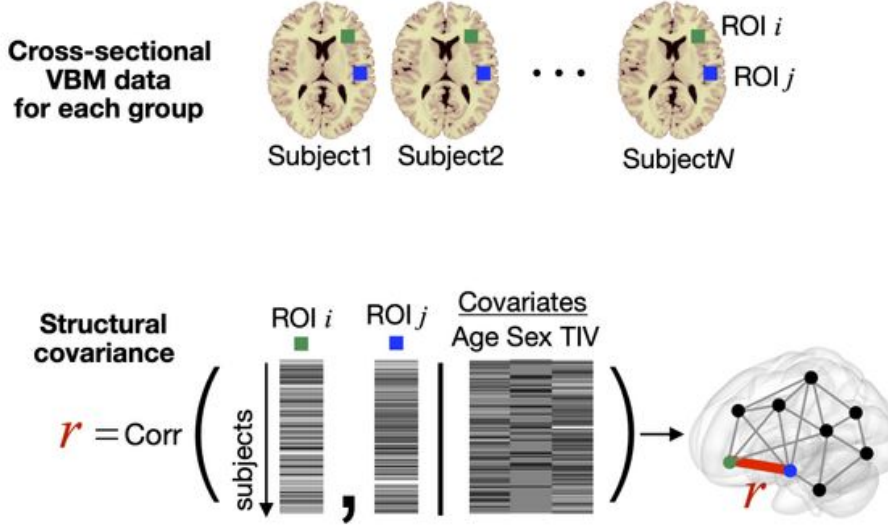

C

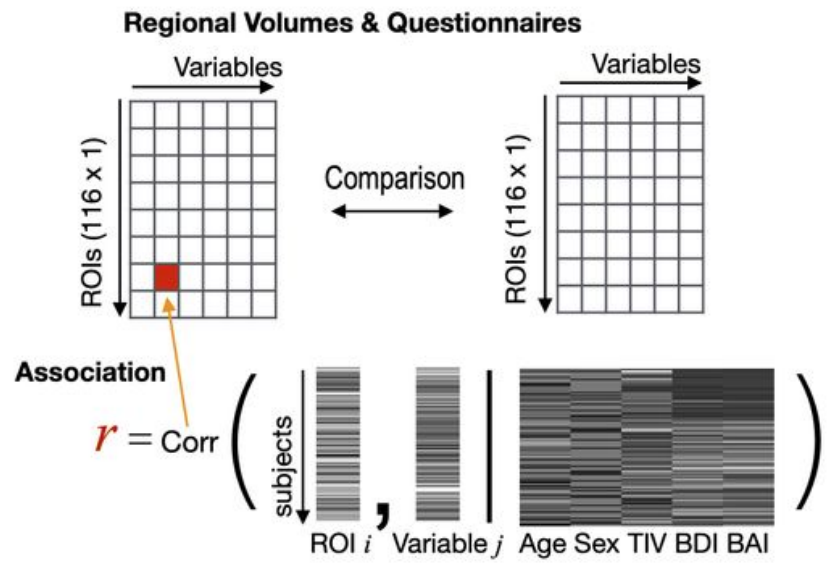

b
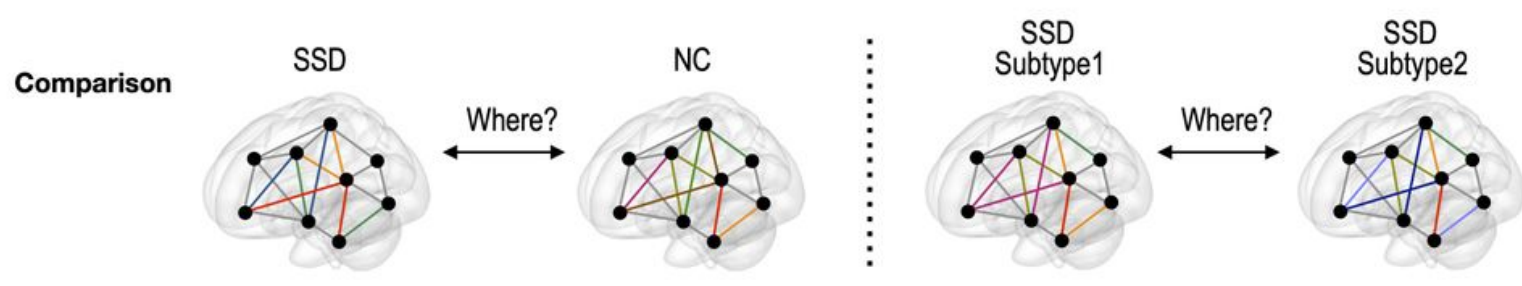

Figure 4

Statistical procedures used in this study. (a) Structural covariance between two regions was defined as partial correlation between two regional volumes (covariates: age, sex, and TIV), which were obtained from cross-sectional VBM data for each group. (b)Structural covariance was compared edge-by-edge between the entire SSD and normal control groups and between the subtype 1 and subtype 2 groups within SSD. (c) Associations between clinical/neurocognitive variables and rGMVs were analyzed. TIV total intra-cranial volume; VBM voxel-based morphometry; SSD somatic symptom disorder; NC, normal control; ROI, region of interest; BDI-II, Beck Depression Inventory-II; BAl, Beck Anxiety Inventory.

\section{Supplementary Files}

This is a list of supplementary files associated with this preprint. Click to download.

- SSDSupplementarytable.doc 